VoL. $73(2006) \quad[335-344]$

\title{
LINEAR STRUCTURE OF WEIGHTED HOLOMORPHIC NON-EXTENDIBILITY
}

\section{BERnAL-GoNZÁLEZ}

In this paper, it is proved that, for any domain $G$ of the complex plane, there exists an infinite-dimensional closed linear submanifold $M_{1}$ and a dense linear submanifold $M_{2}$ with maximal algebraic dimension in the space $H(G)$ of holomorphic functions on $G$ such that $G$ is the domain of holomorphy of every nonzero member $f$ of $M_{1}$ or $M_{2}$ and, in addition, the growth of $f$ near each boundary point is as fast as prescribed.

\section{INTRODUCTION AND NOTATION}

Throughout this paper, the following standard terminology and notation will be used. The symbols $\mathbb{N}, \mathbb{C}, \mathbb{D}, \mathbb{T}$ denote, respectively, the set of positive integers, the complex plane, the open unit disk $\{z \in \mathbb{C}:|z|<1\}$, and the unit circle $\{z \in \mathbb{C}:|z|=1\}$. If $a \in \mathbb{C}$ and $r>0$ then $B(a, r)(\bar{B}(a, r)$, respectively) denotes the open (closed, respectively) Euclidean ball with centre $a$ and radius $r$; in particular, $B(0,1)=\mathbb{D}$. For points $a, b$ of $\mathbb{C}$, the line segment joining $a$ with $b$ is $[a, b]$. If $A \subset \mathbb{C}$ then $\bar{A}\left(A^{0}, \partial A\right.$, respectively) denotes its closure (interior, boundary, respectively) in $\mathbb{C}$. Moreover, if $z_{0} \in \mathbb{C}$ then $d\left(z_{0}, A\right):=\inf \left\{\left|z_{0}-z\right|: z \in A\right\}$. A domain is a nonempty open subset of $\mathbb{C}$. If $G$ is a domain, then $H(G)$ denotes the Fréchet space (= completely metrisable locally convex space) of holomorphic functions on $G$, endowed with the topology of uniform convergence on compacta. In particular, $H(G)$ is a Baire space. Finally, if $a \in G$ and $f \in H(G)$ then $\rho(f, a)$ represents the radius of convergence of the Taylor series of $f$ with centre at $a$. It is well known that $\rho(f, a) \geqslant d(a, \partial G)$.

In $1884 \mathrm{Mittag}$-Leffler (see [9, Chapter 10]) discovered that for any domain $G$ there exists a function $f \in H(G)$ having $G$ as its domain of holomorphy. Recall that $G$ is said to be a domain of holomorphy for $f$ if $f$ is holomorphic exactly at $G$, that is, $f \in H(G)$ and $f$ is analytically non-extendible across $\partial G$ or, more precisely, $\rho(f, a)=d(a, \partial G)$ for all $a \in G$. Note that this implies that $f$ has no holomorphic extension on any domain containing $G$ strictly. Both properties are equivalent if, for instance, $G$ is a Jordan domain, but the equivalence is not general (for instance, consider $G:=\mathbb{C} \backslash(-\infty, 0]$ and

Received 24th October, 2005

Research partially supported by Plan Andaluz de Investigación de la Junta de Andalucía FQM-127 and by DGES Grants BFM2003-03893-C02-01 and MTM2004-21420-E.

Copyright Clearance Centre, Inc. Serial-fee code: 0004-9727/06 \$A2.00+0.00. 
$f:=$ the principal branch of the logarithm on $G)$. By $H_{e}(G)$ we denote the subclass of functions which are holomorphic exactly at $G$. Hence, the Mittag-Leffler result mentioned above says that $H_{e}(G) \neq \emptyset$ for any domain $G$.

In 1933 Kierst and Szpilrajn [12] showed that at least for $G=\mathbb{D}$ the property discovered by Mittag-Leffler is generic, in the sense that $H_{e}(\mathbb{D})$ is not only nonempty but even residual -hence dense- in $H(\mathbb{D})$, that is, its complement in $H(\mathbb{D})$ is of first category. Recently, Kahane ([11, Theorem 3.1 and following remarks]; see also [10, Proposition 1.7.6] and [4, Theorem 3.1]) has observed that Kierst-Szpilrajn's theorem can be extended to every domain $G$ and to rather general topological vector spaces $X \subset H(G)$ (including the full space $X=H(G)$ ); indeed, under suitable conditions on $X$, he shows that $H_{e}(G) \cap X$ is residual in $X$. In other words, $H_{e}(G) \cap X$ is topologically large in $X$.

Recently, we have proved [4] for the case $G=\mathbb{D}$ that under adequate hypotheses a topological vector space $X \subset H(\mathbb{D})$ satisfies that $H_{e}(\mathbb{D}) \cap X$ is also algebraically large, in the sense that the last subset contains -except for zero- some "large" (= dense, or closed infinite-dimensional) linear manifold. Again, the case $X=H(\mathbb{D})$ is covered. Note that the fact that $H_{e}(G)$ is not a linear manifold increases the interest in this matter. As for a general domain $G$, Aron, García and Maestre [1, Theorem 8] had already proved in 2001 that $H(G)$ contains a dense linear manifold $M_{1}$ as well as a closed infinite-dimensional linear manifold $M_{2}$ such that $M_{i} \backslash\{0\} \subset H_{e}(G)(i=1,2)$. In fact, their result extends to any domain of holomorphy in $\mathbb{C}^{N}$ (see also $[4$, Theorem 5.1] for an independent, different proof in the 'dense' case with $N=1)$, and the manifolds $M_{i}(i=1,2)$ are even ideals.

In the terminology of [8], a subset $S$ of a linear topological space $E$ is spaceable whenever $S \cup\{0\}$ contains some closed infinite-dimensional subspace in $E$ (see [8] and [2] for nice, recent examples of spaceable sets). Therefore, under this convention, it has been demonstrated in [1, Theorem 8] that $H_{e}(G)$ is spaceable in $H(G)$.

Nevertheless, the approach in [1, Theorem 8] does not give any information about how fast the functions in $M_{1}$ or $M_{2}$ can grow near the boundary. In [4, note after Theorem $5.1]$ it is suggested how this can be proved for the manifold $M_{1}$ ('dense' case) in $H(G)$, with $G \subset \mathbb{C}$. Hence, it is natural to ask the following:

Given any prescribed ('weight') function $\varphi: G \rightarrow(0,+\infty)$, is the set

$$
\mathcal{S}_{\varphi}:=\left\{f \in H_{e}(G): \limsup _{z \rightarrow t}|f(z)| / \varphi(z)=+\infty \text { for all } t \in \partial G\right\}
$$

spaceable in $H(G)$ ?

The main aim in this paper is to furnish an affirmative answer to this question. This will be obtained in Section 2. Finally, in Section 3 we shall complete this study by showing the existence of a dense linear submanifold $M$ with maximal algebraic dimension -that is, $\operatorname{dim}(M)=\chi:=$ the cardinality of the continuum- such that $M \backslash\{0\} \subset \mathcal{S}_{\varphi}$, where $\varphi$ is a given weight function as above. 


\section{SPACEABILITY OF THE WEIGHTED NON-EXTENDIBILITY}

Before establishing our main result, an auxiliary statement about basic sequences is needed. Let us consider the Hilbert space $L^{2}(\mathbb{T})$ of all (Lebesgue classes of) measurable functions $f: \mathbb{T} \rightarrow \mathbb{C}$ with finite quadratic norm

$$
\|f\|_{2}:=\left(\int_{0}^{2 \pi}\left|f\left(e^{i \theta}\right)\right|^{2} d \theta /(2 \pi)\right)^{1 / 2} .
$$

Since $\left(z^{n}\right)_{n=-\infty}^{\infty}$ is an orthonormal basis of $L^{2}(\mathbb{T})$, we have that $\left(z^{n}\right)_{n \geqslant 1}$ is a basic sequence in $L^{2}(\mathbb{T})$. Recall that two basic sequences $\left(x_{n}\right)_{n \geqslant 1},\left(y_{n}\right)_{n \geqslant 1}$ in a Banach space $(E,\|\cdot\|)$ are said to be equivalent if, for every sequence $\left(a_{n}\right)_{n \geqslant 1}$ of scalars, the series $\sum_{n=1}^{\infty} a_{n} x_{n}$ converges if and only if the series $\sum_{n=1}^{\infty} a_{n} y_{n}$ converges. This happens (see [3, p. 108]) if and only if there exist two constants $m, M \in(0,+\infty)$ such that, for every finite sequence $\left(a_{j}\right)_{j=1, \ldots, J}$ of scalars, we have

$$
m\left\|\sum_{j=1}^{J} a_{j} x_{j}\right\| \leqslant\left\|\sum_{j=1}^{J} a_{j} y_{j}\right\| \leqslant M\left\|\sum_{j=1}^{J} a_{j} x_{j}\right\| .
$$

LEMMA 2.1. Assume that $G$ is a domain with $\overline{\mathbb{D}} \subset G$ and that $\left(f_{j}\right)_{j \geqslant 1} \subset H(G)$ is a sequence such that it is a basic sequence in $L^{2}(\mathbb{T})$ that is equivalent to $\left(z^{j}\right)_{j \geqslant 1}$. If

$$
\left\{h_{l}:=\sum_{j=1}^{J(l)} c_{j, l} f_{j}\right\}_{l \geqslant 1}
$$

is a sequence in $\operatorname{span}\left(f_{j}\right)_{j \geqslant 1}$ converging in $H(G)$, then

$$
\sup _{l \in \mathrm{N}} \sum_{j=1}^{J(l)}\left|c_{j, l}\right|^{2}<+\infty
$$

Proof: Observe first that, since $\overline{\mathbb{D}}$ is a compact subset of $G$, convergence in $H(G)$ is stronger than convergence in $L^{2}(\mathbb{T})$-norm. Therefore $\left(h_{l}\right)_{l \geqslant 1}$ converges in $L^{2}(\mathbb{T})$, so the sequence $\left(\left\|h_{l}\right\|_{2}\right)_{l \geqslant 1}$ is bounded, say $\left\|h_{l}\right\|_{2} \leqslant \alpha(l \in \mathbb{N})$. Let $x_{j}, y_{j},\|\cdot\|$ be respectively the function $z \mapsto z^{j}$, the function $f_{j}$ and the norm $\|\cdot\|_{2}$. Then, by (1), we get for every $l \in \mathbb{N}$ that

$$
m^{2} \sum_{j=1}^{J(l)}\left|c_{j, l}\right|^{2}=m^{2}\left\|\sum_{j=1}^{J(l)} c_{j, l} z^{j}\right\|_{2}^{2} \leqslant\left\|\sum_{j=1}^{J(l)} c_{j, l} f_{j}\right\|_{2}^{2}=\left\|h_{l}\right\|_{2}^{2} \leqslant \alpha^{2} .
$$

Hence (2) is satisfied because the supremum is not greater that $\alpha^{2} / \mathrm{m}^{2}$.

Now, our main assertion about non-extendibility can be established. 
THEOREM 2.2. Let $G \subset \mathbb{C}$ be a domain and $\varphi: G \rightarrow(0,+\infty)$ be a function. Then $\mathcal{S}_{\varphi}$ is spaceable in $H(G)$.

Proof: We must prove the existence of an infinite-dimensional closed linear manifold $M$ in $H(G)$ such that $M \backslash\{0\} \subset \mathcal{S}_{\varphi}$. The case $G=\mathbb{C}$ being trivial, we may assume $G \neq \mathbb{C}$. We denote by $G_{*}$ the one-point compactification of $G$. Recall that in $G_{*}$ the whole boundary $\partial G$ collapses to a unique point, say $\omega$. Without loss of generality, it can be supposed that $\overline{\mathbb{D}} \subset G$.

We are going to choose countably many pairwise disjoint sequences $\{a(k, n): n \in \mathbb{N}\}$ $(k \in \mathbb{N}$ ) of distinct points of $G \backslash \overline{\mathbb{D}}$ such that each of them has no accumulation point in $G$ and every prime end (see [5, Chapter 9]) of $\partial G$ is an accumulation point of each such sequence. The last property means, more precisely, the following: For every $k \in \mathbb{N}$, every $a \in G$ and every $r>d(a, \partial G)$, the intersection of $\{a(k, n): n \in \mathbb{N}\}$ with the connected component of $B(a, r) \cap G$ containing $a$ is infinite. In particular, every point $t \in \partial G$ would be an accumulation point of each sequence $\{a(k, n): n \in \mathbb{N}\}$.

Let us show how such a family of sequences can be constructed. We begin with $k=1$. Let $\left\{c_{j}: j \in \mathbb{N}\right\}$ be a dense countable subset of $G$. For each $j \in \mathbb{N}$ choose $b_{j} \in \partial G$ such that $\left|b_{j}-c_{j}\right|=d\left(c_{j}, \partial G\right)$. For every $j \in \mathbb{N}$ let $\left\{d_{1, j, l}: l \in \mathbb{N}\right\}$ be a sequence of points in $\left[c_{j}, b_{j}\right] \backslash \overline{\mathbb{D}}$ such that

$$
\left|d_{1, j, l}-b_{j}\right|<1 /(1+j+l) \quad(j, l \in \mathbb{N}) .
$$

Then we choose as $\{a(1, n): n \in \mathbb{N}\}$ a one-fold sequence (without repetitions) consisting of all distinct points of the set $\left\{d_{1, j, l}: j, l \in \mathbb{N}\right\}$. It is easy to check that $\{a(1, n): n \in \mathbb{N}\}$ satisfies the required property. In a second step that is, for $k=2$ - we can select for every $j \in \mathbb{N}$ a sequence $\left\{d_{2, j, l}: l \in \mathbb{N}\right\}$ of points of $\left[c_{j}, b_{j}\right] \backslash(\overline{\mathbb{D}} \cup\{a(1, n): n \in \mathbb{N}\})$ such that, in addition,

$$
\left|d_{2, j, l}-b_{j}\right|<1 /(2+j+l) \quad(j, l \in \mathbb{N})
$$

this is possible due to the denumerability of $\{a(1, n): n \in \mathbb{N}\}$. Again, we define $\{a(2, n)$ : $n \in \mathbb{N}\}$ as a sequence consisting of all distinct points of the set $\left\{d_{2, j, l}: j, l \in \mathbb{N}\right\}$; it then satisfies the required prime end property. It is now clear that this process can be repeated inductively, so yielding the desired disjoint family

$$
\{\{a(k, n): n \in \mathbb{N}\}: k \in \mathbb{N}\} .
$$

Secondly, let us consider the subset $A:=\overline{\mathbb{D}} \cup B \subset G$, where

$$
B:=\{a(k, n): k, n \in \mathbb{N}\} .
$$

Recall that for each $k \in \mathbb{N}$ the sequence $\{a(k, n): n \in \mathbb{N}\}$ is an enumeration of the distinct points of a certain subset $\left\{d_{k, j, l}: j, l \in \mathbb{N}\right\} \subset G$ satisfying

$$
\left|d_{k, j, l}-b_{j}\right|<\frac{1}{k+j+l}(j, l \in \mathbb{N}) .
$$


We have that $A$ is relatively closed in $G$. Indeed, the set of accumulation points of $A$ in $G$ is just $\overline{\mathbb{D}}$ (which is included in $A$ ) because the set of accumulation points of $B$ in $G$ is empty. Let us explain why this is so. Assume, by way of contradiction, that $z_{0} \in G$ is an accumulation point of $B$. Then there is a sequence of distinct points $\left(d_{k(n), j(n), l(n)}\right)_{n \geqslant 1}$ in $B$ tending to $z_{0}$. Then the set $\{(k(n), j(n), l(n)): n \in \mathbb{N}\}$ is infinite, so at least one of the sets of positive integers $\{k(n): n \in \mathbb{N}\},\{j(n): n \in \mathbb{N}\},\{l(n): n \in \mathbb{N}\}$ is infinite, hence unbounded. Therefore the sequence $(k(n)+j(n)+l(n))_{n \geqslant 1}$ is also unbounded, thus $k(n)+j(n)+l(n)>2 / d\left(z_{0}, \partial G\right)$ for infinitely many $n \in \mathbb{N}$. Consequently,

$$
\begin{aligned}
\left|d_{k(n), j(n), l(n)}-z_{0}\right| & \geqslant\left|z_{0}-b_{j(n)}\right|-\left|d_{k(n), j(n), l(n)}-b_{j(n)}\right| \\
& \geqslant d\left(z_{0}, \partial G\right)-\frac{1}{k(n)+j(n)+l(n)}>\frac{d\left(z_{0}, \partial G\right)}{2}
\end{aligned}
$$

for infinitely many $n \in \mathbb{N}$, which is absurd.

Thus, $A$ is closed in $G$. But note that $G_{*} \backslash A$ is connected as well as locally connected at $\omega$, because $\overline{\mathbb{D}}$ is compact (so it is "far" from $\omega$, and we can suppose that the basic connected neighbourhoods of $\omega$ do not intersect $\overline{\mathbb{D}}), G \backslash \overline{\mathbb{D}}$ is connected and $B$ is countable (so deleting $B$ from $G \backslash \overline{\mathbb{D}}$ makes no influence in connectedness or local connectedness). Let us consider, for every $N \in N$, the function $g_{N}: A \rightarrow \mathbb{C}$ defined as

$$
g_{N}(z)= \begin{cases}z^{N} & \text { if } z \in \overline{\mathbb{D}} \\ n(1+\varphi(a(N, n))) & \text { if } z=a(N, n) \text { and } n \in \mathbb{N}, \\ 0 & \text { if } z=a(k, n) \text { and } k, n \in \mathbb{N} \text { with } k \neq N\end{cases}
$$

Observe that $g_{N}$ is continuous on $A$ and holomorphic on $A^{0}(=\mathbb{D})$. Then the Arakelian approximation theorem (see [7, pp. 136-144]) guarantees the existence of a function $f_{N} \in H(G)$ such that

$$
\left|f_{N}(z)-g_{N}(z)\right|<\frac{1}{3^{N}} \text { for all } z \in A .
$$

Consequently, one obtains

$$
\begin{gathered}
\left|f_{N}(z)-z^{N}\right|<\frac{1}{3^{N}} \text { for all } z \in \overline{\mathbb{D}} \\
\left|f_{N}(a(N, n))-n(1+\varphi(a(N, n)))\right|<1 \text { for all } n \in \mathbb{N} \text {, and } \\
\left|f_{N}(a(k, n))\right|<\frac{1}{3^{N}} \text { for all } n \in \mathbb{N} \text { and all } k \in \mathbb{N} \backslash\{N\}
\end{gathered}
$$

Finally, we define the sought-after linear manifold $M$ by

$$
M:=\operatorname{closure}_{H(G)}\left(\operatorname{span}\left\{f_{N}: N \in \mathbb{N}\right\}\right) .
$$

It is clear that $M$ is a closed linear manifold in $H(G)$. On the other hand, we have from (4) that $\left\|f_{N}-\varphi_{N}\right\|_{2}<3^{-N}$ for all $N \in \mathbb{N}$ (where $\varphi_{N}(z):=z^{N}$ ). By using this last 
inequality as well as the fact $\sum_{N=1}^{\infty} 3^{-N}<1$ together with the basis perturbation theorem [6, p. 46, Theorem 9], we can derive that $\left(f_{N}\right)_{N \geqslant 1}$ is a basic sequence in $L^{2}(\mathbb{T})$. Indeed, let $\left(e_{n}^{*}\right)_{n \geqslant 1}$ be the sequence of coefficient functionals corresponding to the basic sequence $\left(z^{n}\right)_{n \geqslant 1}$. Since $\left\|e_{n}^{*}\right\|_{2}=1(n \in \mathbb{N})$, one obtains

$$
\sum_{N=1}^{\infty}\left\|e_{n}^{*}\right\|_{2}\left\|f_{N}-\varphi_{N}\right\|<1
$$

Therefore the perturbation theorem applies because $\left(\varphi_{N}\right)_{N \geqslant 1}$ is a basic sequence.

Since $\left(f_{N}\right)_{N \geqslant 1}$ is a basic sequence, we get that, in particular, the functions $f_{N}(N$ $\in \mathbb{N})$ are linearly independent. Hence $M$ has infinite dimension.

It remains to show that $M \backslash\{0\} \subset \mathcal{S}_{\varphi}$. Fix $f \in M \backslash\{0\}$. Since the convergence in $H(G)$ is stronger that the convergence in $L^{2}(\mathbb{T}$ ), we have that (the restriction to $\mathbb{T}$ of) $f$ is in $\widetilde{M}:=\operatorname{closure}_{L^{2}(\mathbb{T})}\left(\operatorname{span}\left\{f_{N}: N \in \mathbb{N}\right\}\right)$. Therefore $f$ has a (unique) representation $f=\sum_{j=1}^{\infty} c_{j} f_{j}$ in $L^{2}(\mathbb{T})$, because $\left(f_{N}\right)_{N \geqslant 1}$ is a basic sequence in this space. But $f \neq 0$, so there is $N \in \mathbb{N}$ with $c_{N} \neq 0$. On the other hand, there is a sequence

$$
\left\{h_{l}:=\sum_{j=1}^{J(l)} c_{j, l} f_{j}\right\}_{l \geqslant 1}
$$

in $\operatorname{span}\left\{f_{j}: j \in \mathbb{N}\right\}$ (without loss of generality, we can assume that $J(l) \geqslant N$ for all $l$ ) that converges to $f$ compactly in $G$. By Lemma 2.1,

$$
C:=\sup _{l \in \mathbb{N}} \sum_{j=1}^{J(l)}\left|c_{j, l}\right|^{2}<+\infty .
$$

But $\left(h_{l}\right)_{l \geqslant 1}$ also converges to $f$ in $L^{2}(\mathbb{T})$, so the continuity of each projection

$$
\sum_{j=1}^{\infty} d_{j} f_{j} \in \widetilde{M} \mapsto d_{m} \in \mathbb{C} \quad(m \in \mathbb{N})
$$

yields that $\lim _{l \rightarrow \infty} c_{N, l}=c_{N}$. In particular, there exists $l_{0} \in \mathbb{N}$ such

$$
\left|c_{N, l}\right| \geqslant \frac{\left|c_{N}\right|}{2} \text { for all } l \geqslant l_{0}
$$

Let us fix $n \in \mathbb{N}$. Since the singleton $\{a(N, n)\}$ is a compact subset of $G$, we get the existence of a positive integer $l=l(n) \geqslant l_{0}$ such that

$$
\left|h_{l}(a(N, n))-f(a(N, n))\right|<1 .
$$


By using (5), (6), (7), (8), the triangle inequality and the Cauchy-Schwarz inequality, we obtain

$$
\begin{aligned}
|f(a(N, n))| & \geqslant\left|h_{l}(a(N, n))\right|-1 \\
& \geqslant\left|c_{N, l} f_{N}(a(N, n))\right|-\sum_{\substack{j=1 \\
j \neq N}}^{J(l)}\left|c_{j, l} f_{j}(a(N, n))\right|-1 \\
& \geqslant \frac{\left|c_{N}\right|}{2}(n(1+\varphi(a(N, n)))-1)-\sum_{\substack{j=1 \\
j \neq N}}^{J(l)}\left|c_{j, l}\right| \frac{1}{3^{j}}-1 \\
& \geqslant \frac{\left|c_{N}\right|}{2}(n(1+\varphi(a(N, n)))-1)-\left(\sum_{j=1}^{\infty}\left(\frac{1}{3^{j}}\right)^{2}\right)^{1 / 2}\left(\sum_{\substack{j=1 \\
j \neq N}}^{J(l)}\left|c_{j, l}\right|^{2}\right)^{1 / 2}-1 \\
& \geqslant \frac{\left|c_{N}\right|}{2}(n(1+\varphi(a(N, n)))-1)-C^{1 / 2}-1
\end{aligned}
$$

Consequently,

$$
\lim _{n \rightarrow \infty} f(a(N, n))=\infty=\lim _{n \rightarrow \infty} f(a(N, n)) / \varphi(a(N, n)) .
$$

The second equality shows that $\limsup |f(z)| / \varphi(z)=+\infty$ for all $t \in \partial G$, because each boundary point is a limit point of $\left(z_{n}:=a(N, n)\right)_{n \geqslant 1}$.

Now, it is time to use the prime end approximation property of the sequence $\left(z_{n}\right)$. Suppose, by way of contradiction, that $f \notin \mathcal{S}_{\varphi}$. Then $f \notin H_{e}(G)$, so there must be a point $c \in G$ such that $\rho(f, c)>d(c, \partial G)$. Choose $r$ with $d(c, \partial G)<r<\rho(f, c)$. By the construction of the sequences $(a(k, n))_{n \geqslant 1}(k \in \mathbb{N})$, there exists a sequence $\left\{n_{1}<n_{2}\right.$ $\langle\cdots\} \subset \mathbb{N}$ for which $z_{n_{j}} \in G \cap B(c, r)(j \in \mathbb{N})$. Finally, the sum $S(z)$ of the Taylor series of $f$ with centre $c$ is bounded on $B(c, r)$. But $S=f$ on $G \cap B(c, r)$, so $S\left(z_{n_{j}}\right)$ $=f\left(z_{n_{j}}\right) \rightarrow \infty(j \rightarrow \infty)$, which is absurd. This contradiction finishes the proof.

\section{MANifoldS With MAXIMAL ALGEBRAIC DIMENSION}

We conclude this note with a theorem that completes our Theorem 2.2 as well as Theor $\epsilon . \eta 5.1$ in [4] and (in the one-dimensional case) Theorem 8 in [1]. Specifically, we are able to construct -for a prescribed function $\varphi: G \rightarrow(0,+\infty)$ - a linear submanifold $M \subset H(G)$ with $M \backslash\{0\} \subset \mathcal{S}_{\varphi}$ that is not only dense, but even it satisfies $\operatorname{dim}(M)=\chi$ (notice that the dense linear manifold $M$ whose construction is suggested in [4, note following Theorem 5.1] was only of countably infinite dimension; in the opposite direction, the dense manifold $X$ provided in [1, Theorem 8] does satisfy $\operatorname{dim}(X)=\chi$, but the fact $X \backslash\{0\} \subset \mathcal{S}_{\varphi}$ does not hold). Observe that, as an easy consequence of Baire's 
category theorem and of the fact that $H(G)$ is infinite-dimensional, metrisable, separable and complete, we have $\operatorname{dim}(H(G))=\chi$. Hence $\chi$ is the maximal algebraic dimension which is permitted for the submanifolds of $H(G)$. For instance, the linear manifold $M$ constructed in the proof of Theorem 2.2 satisfies $\operatorname{dim}(M)=\chi$ (because it is a closed subspace of $H(G)$, so $M$ is also infinite-dimensional, metrisable, separable and complete) but it is not dense.

THEOREM 3.1. Let $G \subset \mathbb{C}$ be a domain and $\varphi: G \rightarrow(0,+\infty)$ be a function. Then there is a dense linear manifold $M$ in $H(G)$ such that $\operatorname{dim}(M)=\chi$ and $M \backslash\{0\}$ $\subset \mathcal{S}_{\varphi}$.

Proof: Again, the case $G=\mathbb{C}$ is trivial, so we suppose $G \neq \mathbb{C}$. First, we consider pairwise disjoint sequences $\{a(k, n): n \in \mathbb{N}\}(k \in \mathbb{N})$, and then we select a sequence $\left\{f_{N}: N \in \mathbb{N}\right\} \subset H(G)$. This is made exactly as in the proof of Theorem 2.2, with the sole exception that instead of (5) we have

$$
\left|f_{N}(a(N, n))-n^{1 / 2}(1+\varphi(a(N, n)))\right|<1 \text { for all } n \in \mathbb{N} \text {. }
$$

In other words, with the notation of the proof of Theorem 2.2 we would define

$$
g_{N}(a(N, n)):=n^{1 / 2}(1+\varphi(a(N, n))) \quad(N, n \in \mathbb{N})
$$

before the application of Arakelian's theorem. The key point will be that $n^{1 / 2}$ tends to infinity as $n \rightarrow \infty$, but less rapidly than any power $n^{N}(N \in \mathbb{N})$. Let us define

$$
M_{1}:=\operatorname{closure}_{H(G)}\left(\operatorname{span}\left\{f_{N}: N \in \mathbb{N}\right\}\right) .
$$

Therefore we obtain as in the proof of Theorem 2.2 that $M_{1} \backslash\{0\} \subset \mathcal{S}_{\varphi}$. As observed at the beginning of this section, we have $\operatorname{dim}\left(M_{1}\right)=\chi$.

Second, fix an increasing sequence $\left\{K_{n}: n \in \mathbb{N}\right\}$ of compact subsets of $G$ such that each compact subset of $G$ is contained in some $K_{n}$ and each component of the complement of every $K_{n}$ contains some connected component of the complement of $G$ (see [13, Chapter 13]). Choose a dense countable subset $\left\{\psi_{n}: n \in \mathbb{N}\right\}$ of $H(G)$. Now consider for each $N \in \mathbb{N}$ the set $A_{N}:=K_{N} \cup\{a(k, n): k, n \in \mathbb{N}\}$. In a similar way to the proof of [4, Theorem 5.2], we have that $A_{N}$ is closed in $G$ and that $G_{*} \backslash A_{N}$ is connected and locally connected at $\omega$. The function $h_{N}: A_{N} \rightarrow \mathbb{C}$ defined as

$$
h_{N}(z)= \begin{cases}\psi_{N}(z) & \text { if } z \in K_{N}, \\ n^{N}(1+\varphi(a(k, n))) & \text { if } z=a(k, n)(k, n \in \mathbb{N}) \text { and } z \notin K_{N}\end{cases}
$$

is continuous on $A_{N}$ and holomorphic on $A_{N}^{0}\left(=K_{N}^{0}\right)$. We now use again the Arakelian approximation theorem to obtain this time a function $F_{N} \in H(G)$ such that

$$
\left|F_{N}(z)-h_{N}(z)\right|<\frac{1}{N} \text { for all } z \in A_{N} .
$$


From (10) we derive that $\left|F_{N}(z)-\psi_{N}(z)\right|<1 / N$ for all $z \in A_{N}$ and all $N \in \mathbb{N}$. These inequalities together with the denseness of $\left\{\psi_{N}: N \in \mathbb{N}\right\}$ and the exhaustion property of the family $\left\{K_{N}: N \in \mathbb{N}\right\}$ yield the denseness of the sequence $\left\{F_{N}: N \in \mathbb{N}\right\}$ in $H(G)$.

Finally, we define $M$ as

$$
M:=\operatorname{span}\left(M_{1} \cup\left\{F_{N}: N \in \mathbb{N}\right\}\right) .
$$

Since $M \supset\left\{F_{N}: N \in \mathbb{N}\right\}$ and $M \supset M_{1}$, it is evident that $M$ is a dense linear submanifold of $H(G)$ and $\operatorname{dim}(M)=\chi$. It remains to show that $M \backslash\{0\} \subset \mathcal{S}_{\varphi}$. For this, fix a function $f \in M \backslash\{0\}$. If $f \in M_{1}$ then we already know that $f \in \mathcal{S}_{\varphi}$. Thus, we can assume that $f \in M \backslash M_{1}$. Then there are finitely many scalars $c_{1}, \ldots, c_{N}, d_{1}, \ldots, d_{\mu}$ with $c_{N} \neq 0$ such that

$$
f=\sum_{j=1}^{N} c_{j} F_{j}+\sum_{j=1}^{\mu} d_{j} f_{j} .
$$

Recall that according to the proof of Theorem 2.2 the set $B:=\{a(k, n): k, n \in \mathbb{N}\}$ has no accumulation point in $G$. In particular, each compact set $K_{j}$ may contain only finitely many points $a(k, n)$. Therefore we can derive from (10) the existence of a number $n_{0} \in \mathbb{N}$ such that

$$
\left|F_{j}(a(N, n))-n^{j}(1+\varphi(a(N, n)))\right|<1 \text { for all } n \geqslant n_{0}(j=1, \ldots, N) .
$$

On the other hand, we obtain by (6) and (9) that

$$
\left|f_{j}(a(N, n))\right|<n^{1 / 2}(1+\varphi(a(N, n)))+1 \quad(j=1, \ldots, \mu ; n \in \mathbb{N}) .
$$

To finish, from (11), (12), (13) and the triangle inequality it is deduced for $n \geqslant n_{0}$ that

$$
\begin{aligned}
|f(a(N, n))| \geqslant\left|c_{N}\right|\left[n^{N}(1+\varphi(a(N, n)))-1\right] & -\sum_{j=1}^{N-1}\left|c_{j}\right|\left[n^{j}(1+\varphi(a(N, n)))+1\right] \\
& -\left(\sum_{j=1}^{\mu}\left|d_{j}\right|\right)\left[n^{1 / 2}(1+\varphi(a(N, n)))+1\right] .
\end{aligned}
$$

Consequently,

$$
\lim _{n \rightarrow \infty} f(a(N, n))=\infty=\lim _{n \rightarrow \infty} f(a(N, n)) / \varphi(a(N, n)) .
$$

Then the desired conclusion may be achieved as in the last paragraph of the proof of Theorem 2.2 .

FinAl QUESTION. Do the analogues of Theorems 2.2 and 3.1 hold for a domain of holomorphy in $\mathbb{C}^{N}$ ? 


\section{REFERENCES}

[1] R. Aron, D. García and M. Maestre, 'Linearity in non-linear problems', Rev. R. Acad. Cien. Exactas. Fis. Nat Ser. A Mat. 95 (2001), 7-12.

[2] F. Bayart, 'Linearity of sets of strange functions', Michigan Math. 53 (2005), 291-303.

[3] B. Beauzamy, Introduction to Banach spaces and their geometry (North Holland, Amsterdam, 1982).

[4] L. Bernal-González, 'Linear Kierst-Szpilrajn theorems', Studia Math. 166 (2005), 55-69.

[5] E.F. Collingwood and A.J. Lohwater, The theory of cluster sets (Cambridge University Press, Cambridge, 1966).

[6] J. Diestel, Sequences and series in Banach spaces (Springer-Verlag, New York, 1984).

[7] D. Gaier, Lectures on complex aproximation (Birkhäuser, Basel, London, Stutgart, 1987).

[8] V. Gurariy and L. Quarta, 'On lineability of sets of continuous functions', J. Math. Anal. Appl. 294 (2004), 62-72.

[9] E. Hille, Analytic function theory, II (Chelsea Publishing Company, New York, 1987).

[10] M. Jarnicki and P. Pflug, Extension of holomorphic functions, Expositions in Mathematics 34 (de Gruyter, Berlin, 2000).

[11] J.P. Kahane, 'Baire's category theorem and trigonometric series', J. Analyse Math. 80 (2000), 143-182.

[12] S. Kierst and E. Szpilrajn, 'Sur certaines singularités des fonctions analytiques uniformes', Fund. Math. 21 (1933), 267-294.

[13] W. Rudin, Real and complex analysis ( $3^{\text {rd }}$ edition) (McGraw-Hill, New York, St. Louis, San Francisco, 1987).

Departamento de Análisis Matemático

Facultad de Matemáticas, Apdo. 1160

Avda. Reina Mercedes

41080 Sevilla

Spain

e-mail: Ibernal@us.es 